\title{
Tuning Organogel and Mesophases with Phenanthroline Ligands and their Copper Complexes by Inter- to Intra-molecular Hydrogen Bonds
}

Raymond Ziessel ${ }^{\dagger} * *$ Guillaume Pickaert, ${ }^{\dagger}$ Franck Camerel, ${ }^{\dagger}$ Bertrand Donnio, ${ }^{ \pm}$Daniel Guillon, ${ }^{ \pm}$Michèle Cesario, ${ }^{\ddagger}$ and Thierry Prangé ${ }^{\text {II }}$

\section{Supporting Information (15 pages)}

Table S1. Summary of crystal data, intensity measurements and structure refinement for ligand $\mathrm{L}^{1}$.

\begin{tabular}{|c|c|}
\hline Formula & C40 H36 N4 O8 \\
\hline Mol wt & 700.73 \\
\hline Cryst syst & triclinic \\
\hline Space group & P-1 \\
\hline$a, \AA$ & $8.974(2)$ \\
\hline$b, \AA$ & $12.086(2)$ \\
\hline$c, \AA$ & $17.068(3)$ \\
\hline$\alpha, \operatorname{deg}$ & $68.88(5)$ \\
\hline$\beta, \operatorname{deg}$ & $80.04(5)$ \\
\hline$\gamma, \operatorname{deg}$ & $79.18(5)$ \\
\hline$V, \AA 3$ & $1684.8(5)$ \\
\hline$Z$ & 2 \\
\hline$F(000)$ & 736 \\
\hline$D c / \mathrm{Mg} \mathrm{m}^{-3}$ & 1.381 \\
\hline$\mu(\lambda=0.966), \mathrm{mm}-1$ & 0.097 \\
\hline Cryst size, $\mathrm{mm}$ & $0.1 \times 0.05 \times 0.025$ \\
\hline Temp, K & $100(2)$ \\
\hline No. of reflns collected & 12525 \\
\hline$\theta$ range,deg & $4.28<2 \theta<29.90$ \\
\hline \multirow[t]{3}{*}{$h k l$ ranges } & $-9 \leq \mathrm{h} \leq 9$ \\
\hline & $-11 \leq \mathrm{k} \leq 12$ \\
\hline & $0 \leq 1 \leq 17$ \\
\hline no. of unique reflns & 3506 \\
\hline no. of parameters / restraints & $475 / 0$ \\
\hline$R 1[\mathrm{I}>2 \sigma(\mathrm{I})]^{\mathrm{a}}$ & 0.0556 \\
\hline weight & $\left.1 /\left[\sigma^{2}\left(\mathrm{Fo}^{2}\right)+(0.0879) \mathrm{P}\right)^{2}+2.05\right]^{\mathrm{b}}$ \\
\hline $\mathrm{w} R 2^{\mathrm{c}}$ & 0.1465 \\
\hline max Fourier diff,e/ $/ \AA^{3}$ & $0.5,-0.2$ \\
\hline
\end{tabular}


TableS2. Selected hydrogen bond lengths ( $(\AA)$ for the free ligand $L^{1}$.

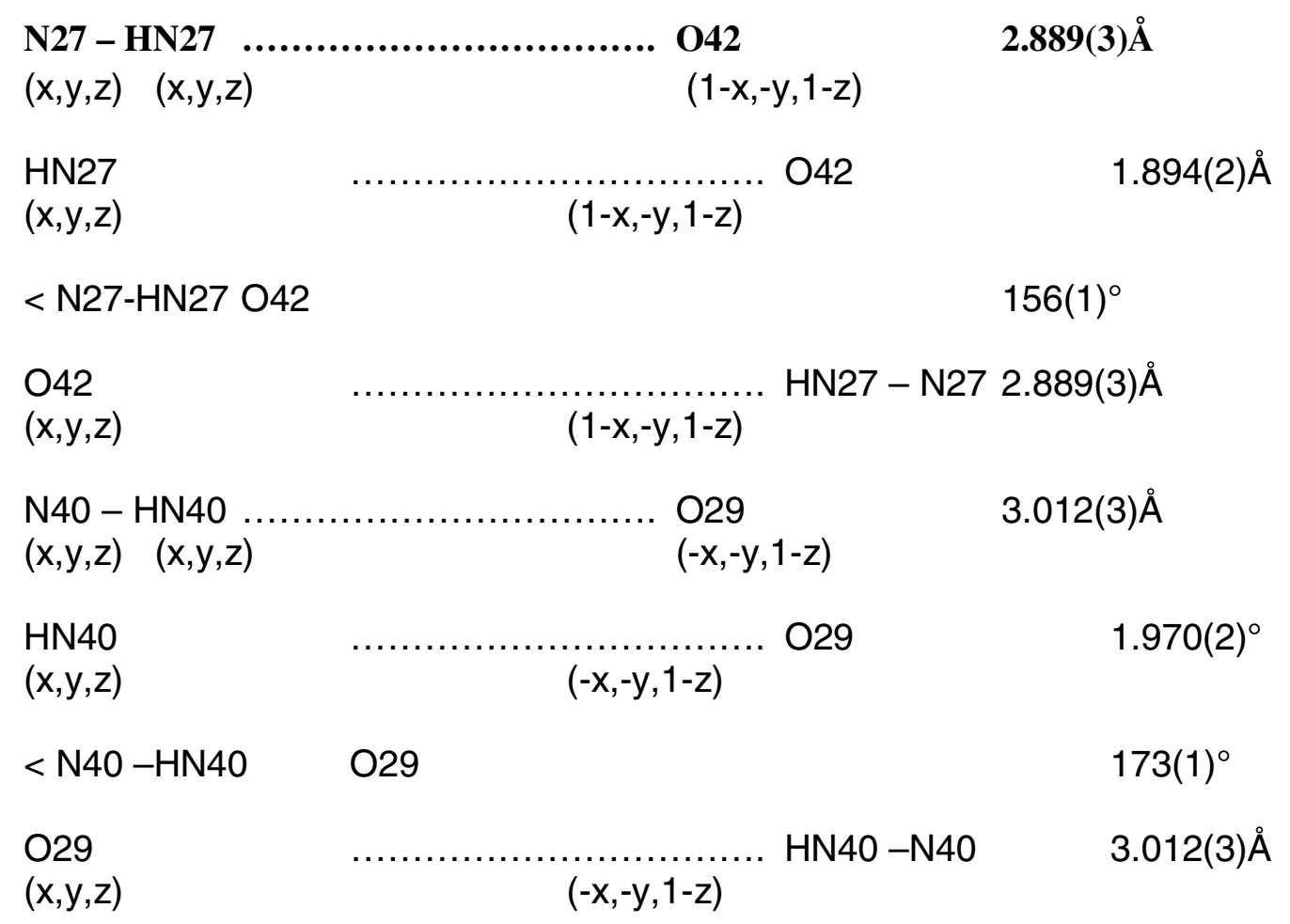




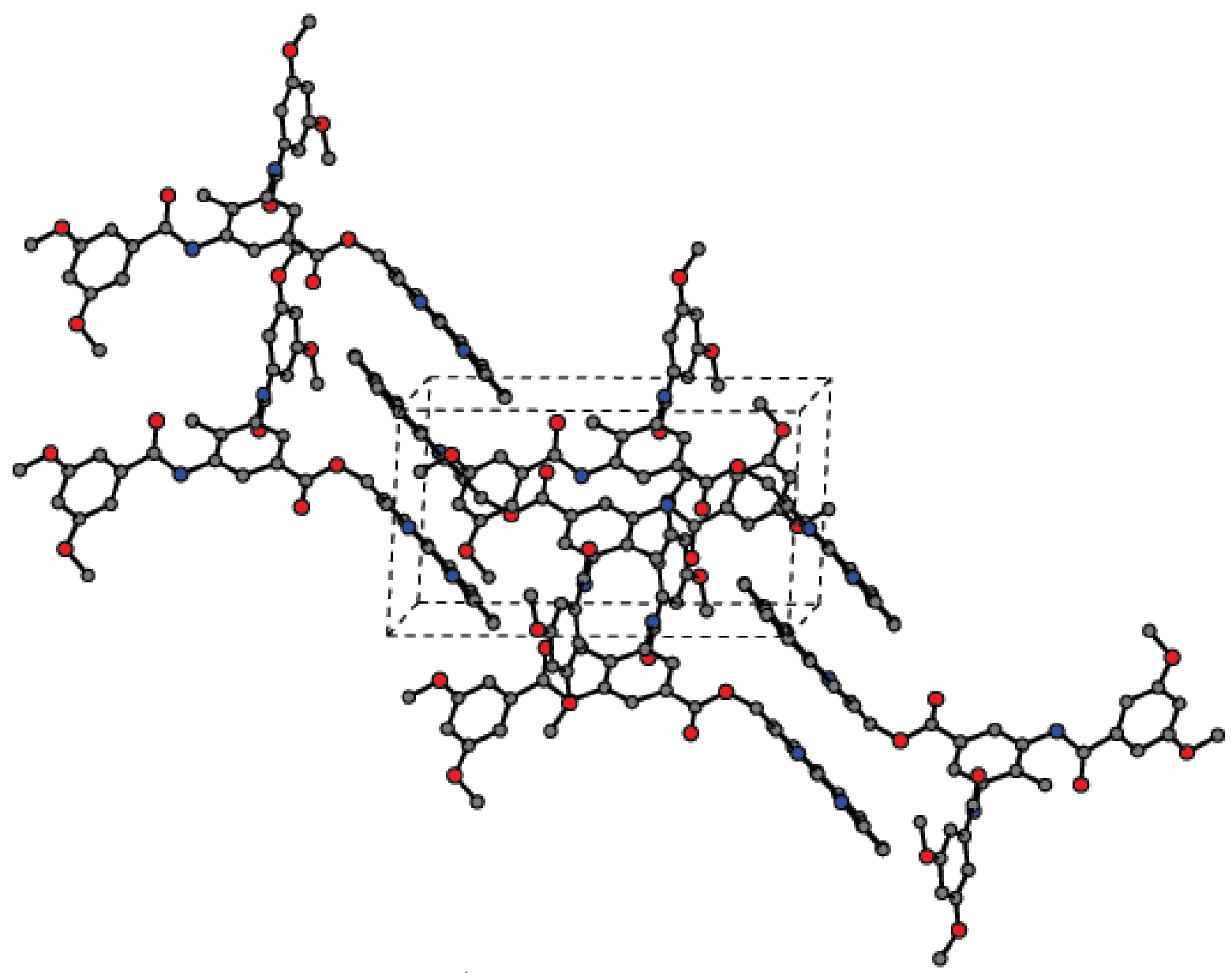

Figure S1. Molecular structure of $L^{1}$ showing the display of the phenyl $\pi$ - $\pi$ stacking (3.5 - 3.6 $\AA$ ) involved in a set of three molecules connected by hydrogen bonds. The phen subunits are perpendicular to this network Schematic representation of the interdigitated phen subunits. 
Table S3. Summary of crystal data, intensity measurements and structure refinement for the $\left[\mathrm{Cu}\left(\mathrm{L}^{1}\right)_{2}\right] \mathrm{ClO}_{4}$ complex.

\begin{tabular}{|c|c|}
\hline Formula & {$\left[\mathrm{CuC}_{80} \mathrm{O}_{16} \mathrm{~N}_{8} \mathrm{H}_{72}\right]^{+},\left(\mathrm{ClO}_{4}\right)^{-}, 4\left(\mathrm{CH}_{3} \mathrm{CN}\right)$} \\
\hline Mol wt & 1728.66 \\
\hline Cryst syst & triclinic \\
\hline Space group & P-1 \\
\hline$a, \AA$ & $15.890(3)$ \\
\hline$b, \AA$ & $16.327(3)$ \\
\hline$c, \AA$ & $17.777(3)$ \\
\hline$\alpha, \operatorname{deg}$ & $79.50(5)$ \\
\hline$\beta, \operatorname{deg}$ & $72.02(5)$ \\
\hline$\gamma, \mathrm{deg}$ & $73.90(5)$ \\
\hline$V, \AA 3$ & $4191.0(13)$ \\
\hline Z & 2 \\
\hline$F(000)$ & 1804 \\
\hline$D \mathrm{c} / \mathrm{Mg} \mathrm{m}^{-3}$ & 1.370 \\
\hline$\mu(\lambda=0.947), \mathrm{mm}-1$ & 0.373 \\
\hline Cryst size, $\mathrm{mm}$ & $0.15 \times 0.05 \times 0.05$ \\
\hline Temp, K & $177(2)$ \\
\hline No. of reflns collected & 7059 \\
\hline$\theta$ range,deg & $2.87<2 \theta<27.95$ \\
\hline \multirow[t]{3}{*}{$h k l$ ranges } & $0 \leq \mathrm{h} \leq 15$ \\
\hline & $-14 \leq \mathrm{k} \leq 15$ \\
\hline & $-15 \leq 1 \leq 16$ \\
\hline no. of unique reflns & 7059 \\
\hline no. of reflns obsd $\mathrm{I}>2 \sigma(\mathrm{I})$ & 5965 \\
\hline no. of parameters / restraints & $675 / 0$ \\
\hline$R 1[\mathrm{I}>2 \sigma(\mathrm{I})]^{\mathrm{a}}$ & 0.1011 \\
\hline weight & $\left.1 /\left[\sigma^{2}\left(\mathrm{Fo}^{2}\right)+(0.1615) \mathrm{P}\right)^{2}+24.20 \mathrm{P}\right]^{\mathrm{b}}$ \\
\hline $\mathrm{w} R 2^{\mathrm{c}}$ & 0.26 \\
\hline max Fourier diff,e/ $/ \AA^{3}$ & $0.79,-0.65$ \\
\hline
\end{tabular}

Table S4. Selected bond lengths $(\AA)$ and angles $\left(^{\circ}\right)$ for the $\left[\mathrm{Cu}\left(\mathrm{L}^{1}\right)_{2}\right] \mathrm{ClO}_{4}$ complex. .

$\begin{array}{lr}\mathrm{Cu}-\mathrm{N}(1 \mathrm{~A}) & 2.021(7) \\ \mathrm{Cu}-\mathrm{N}(1 \mathrm{~B}) & 2.045(7) \\ \mathrm{Cu}-\mathrm{N}(12 \mathrm{~B}) & 2.069(7) \\ \mathrm{Cu}-\mathrm{N}(12 \mathrm{~A}) & 2.075(7) \\ & \\ \mathrm{N}(1 \mathrm{~A})-\mathrm{Cu}-\mathrm{N}(1 \mathrm{~B}) & 135.3(3) \\ \mathrm{N}(1 \mathrm{~A})-\mathrm{Cu}-\mathrm{N}(12 \mathrm{~B}) & 124.2(3) \\ \mathrm{N}(1 \mathrm{~B})-\mathrm{Cu}-\mathrm{N}(12 \mathrm{~B}) & 83.1(3) \\ \mathrm{N}(1 \mathrm{~A})-\mathrm{Cu}-\mathrm{N}(12 \mathrm{~A}) & 82.0(3) \\ \mathrm{N}(1 \mathrm{~B})-\mathrm{Cu}-\mathrm{N}(12 \mathrm{~A}) & 119.0(3) \\ \mathrm{N}(12 \mathrm{~B})-\mathrm{Cu}-\mathrm{N}(12 \mathrm{~A}) & 117.2(3)\end{array}$


Table S5 : Gelation tests and minimum gel concentration $\left(\mathrm{g} \mathrm{L}^{-1}\right)$

\begin{tabular}{|c|c|c|c|c|}
\hline & $\mathbf{L}^{\mathbf{1 2}}$ & $\mathbf{L}^{\mathbf{1 6}}$ & {$\left[\mathbf{C u}\left(\mathbf{L}^{\mathbf{1 2}}\right)_{\mathbf{2}}\right] \mathbf{B F} \mathbf{F}_{\mathbf{4}}$} & {$\left[\mathbf{C u}\left(\mathbf{L}^{\mathbf{1 6}}\right)_{2}\right] \mathbf{B F} \mathbf{F}_{\mathbf{4}}$} \\
\hline Water & Insoluble & Insoluble & & \\
\hline Dimethylformamide & Crystallization & Crystallization & & \\
\hline Hexane & Gel (7) & Gel (1) & Solution & Solution \\
\hline Cyclohexane & Gel (1) & Gel (0.9) & Solution & Solution \\
\hline Dodecane & Gel (3) & Gel (8) & Solution & Solution \\
\hline Dodecanol & Crystallization & Crystallization & & \\
\hline Toluene & Solution & Solution & & \\
\hline Methanol & Insoluble & Insoluble & & \\
\hline
\end{tabular}


Figure S2. XRD diffraction patterns of $\mathrm{L}^{12}$ recorded at $373 \mathrm{~K}$.

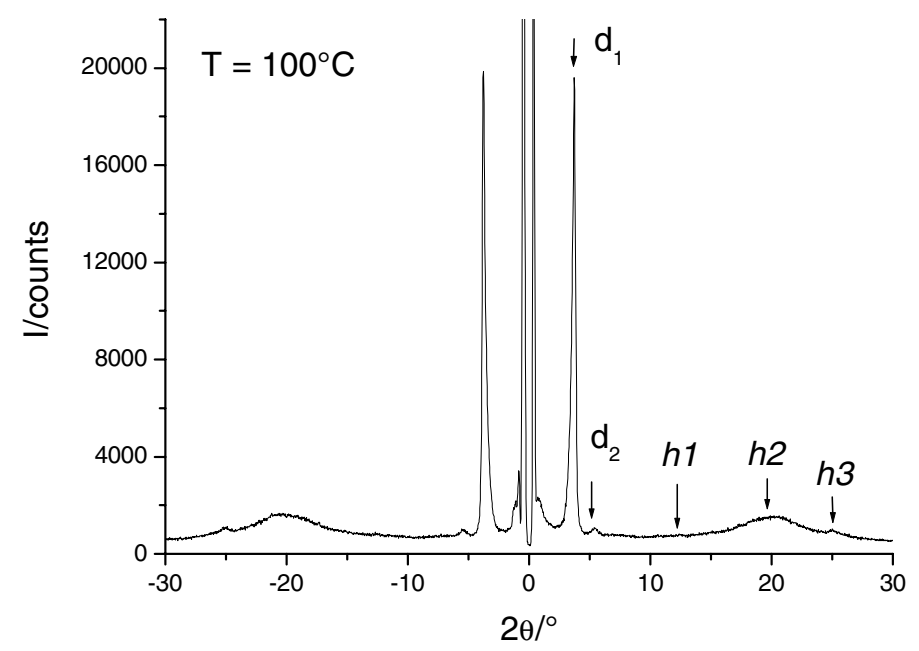

Figure S3. XRD diffraction patterns of $\mathrm{L}^{16}$ recorded at $383 \mathrm{~K}$.

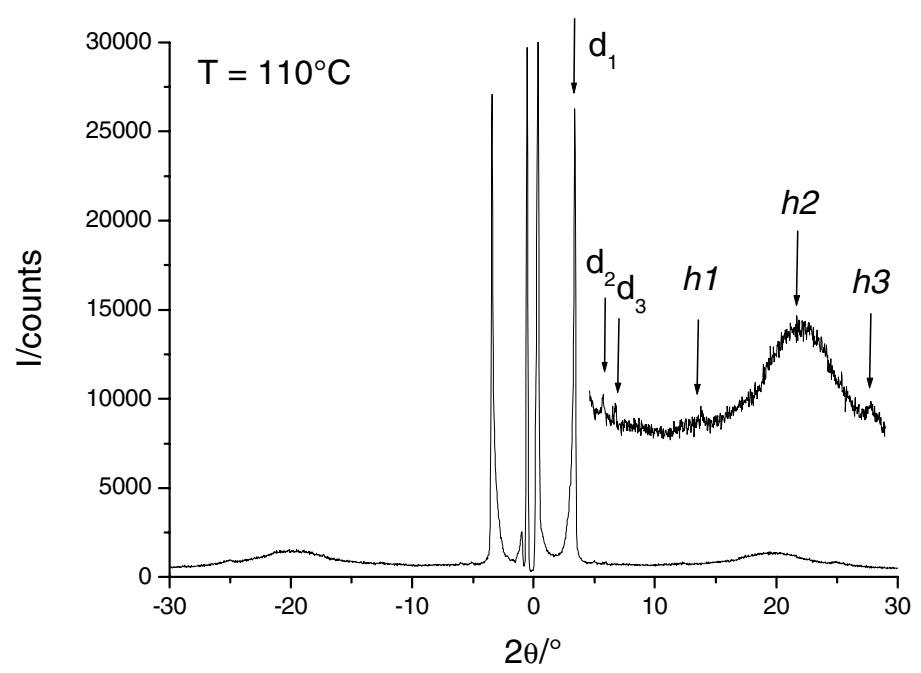

\section{Experimental Section}

General procedure for the preparation of the ligand $\mathbf{L}^{\mathbf{n}}$. A Schlenk flask equipped with a septum and an argon inlet was charged with the acid $\mathbf{1}^{\mathbf{n}}$, distilled $\mathrm{CH}_{2} \mathrm{Cl}_{2}$ (mixture of $\mathrm{CH}_{2} \mathrm{Cl}_{2} / \mathrm{THF}$ for $\mathbf{1}^{\mathbf{1}}$ ), DMAP (1 equiv) and the mixture was stirred until complete solubilization of the acid. Then, $\mathrm{EDC} \cdot \mathrm{HCl}$ (2 equiv) and 2-(hydroxymethyl)-9-methyl-1,10- 
phenantroline ( 1 equiv) were added to the solution, which was stirred overnight. After evaporation of the solvent the purification was performed by flash chromatography on silica gel with $\mathrm{CH}_{2} \mathrm{Cl}_{2} /$ methanol (100/0 to 99/1) as eluant and followed by crystallisation from $\mathrm{CH}_{2} \mathrm{Cl}_{2} / \mathrm{CH}_{3} \mathrm{CN}$.

Ligand $\mathbf{L}^{\mathbf{1}}$. The compound was prepared from $0.100 \mathrm{~g}(0.202 \mathrm{mmol})$ of $\mathbf{1}^{\mathbf{1}}, 0.041 \mathrm{~g}(0.184$ mmol) of 2-(hydroxymethyl)-9-methyl-1,10-phenanthroline, $0.077 \mathrm{~g}$ (0.404 mmol) of $\mathrm{EDC} \cdot \mathrm{HCl}, 0.050 \mathrm{~g}(0.404 \mathrm{mmol})$ of DMAP and distilled $\mathrm{CH}_{2} \mathrm{Cl}_{2} / \mathrm{THF}(20 \mathrm{ml} / 20 \mathrm{ml})$ to afford $0.074 \mathrm{~g}$ of $\mathbf{L}^{1}$ as a white pwder (57\%). ${ }^{1} \mathrm{H}$ NMR (DMSO) $\delta 2.19\left(\mathrm{~s}, 3 \mathrm{H}, \mathrm{CH}_{3}\right), 2.78(\mathrm{~s}, 3 \mathrm{H}$, $\mathrm{CH}_{3}$ phen), $3.81\left(\mathrm{~s}, 12 \mathrm{H}, 4 \mathrm{OCH}_{3}\right), 5.75\left(\mathrm{~s}, 2 \mathrm{H}, \mathrm{OCH}_{2}\right.$ phen), $6.72\left(\mathrm{t},{ }^{4} \mathrm{~J}=2.1 \mathrm{~Hz}, 2 \mathrm{H}, \mathrm{H}\right.$ arom.), 7.17 (d, ${ }^{4} J=2.1 \mathrm{~Hz}, 4 \mathrm{H}, \mathrm{H}$ arom.), 7.66 (d, ${ }^{3} J=8.3 \mathrm{~Hz}, 1 \mathrm{H}, \mathrm{H}$ phen), 7.85 (d, ${ }^{3} J=8.3$ $\mathrm{Hz}, 1 \mathrm{H}, \mathrm{H}$ phen), 7.94 (d, ${ }^{4} \mathrm{~J}=1.9 \mathrm{~Hz}, 2 \mathrm{H}, \mathrm{H}$ phen), 7.97 (s, 2H, H arom), 8.38 (d, ${ }^{3} J=8.0$ $\mathrm{Hz}, 1 \mathrm{H}, \mathrm{H}$ phen), 8.53 (d, ${ }^{3} \mathrm{~J}=8.3 \mathrm{~Hz}, 1 \mathrm{H}, \mathrm{H}$ phen), 8.68 (s, 2H, NH). ${ }^{13} \mathrm{C}$ NMR $(400 \mathrm{MHz}$, DMSO) $\delta$ 14.9, $25.9\left(\mathrm{CH}_{3}\right), 56.4\left(\mathrm{OCH}_{3}\right), 68.8\left(\mathrm{OCH}_{2}\right.$ phen $), 104.5,106.5,121.9,124.6$, $126.2,126.2,127.6,127.7,128.8,137.0,137.3,138.0,138.2,138.4,145.5,156.5,159.5$ (phenyl and phen), 161.3, 166.0 (amide and ester). $\mathrm{FAB}^{+} \mathrm{m} / z$ (nature of the peak, relative intensity) $701.2\left([\mathrm{M}+\mathrm{H}]^{+}, 100\right)$. FT-IR (KBr cell, $\left.\mathrm{cm}^{-1}\right) 3400(\mathrm{sh}), 3232(\mathrm{~m}), 2940$ (m), 2836 (m), 2358 (w), 1718 (m), 1649 (s), 1593 (vs), 1535 (sh), 1510 (s), 1456 (m), 1427 (m), 1350 (m), 1319 (s), 1211 (vs), 1157 (s), 1066 (m). Anal. Calc. for $\mathrm{C}_{40} \mathrm{H}_{36} \mathrm{~N}_{4} \mathrm{O}_{8}$ : C, 68.56; H, 5.18; N, 8.00. Found: C, 68.37; H, 5.08; N, 7.83.

Ligand $\mathbf{L}^{\mathbf{8}}$. The compound was prepared from $0.100 \mathrm{~g}(0.113 \mathrm{mmol})$ of $\mathbf{1}^{\mathbf{8}}, 0.025 \mathrm{~g}(0.113$ mmol) of 2-(hydroxymethyl)-9-methyl-1,10-phenanthroline, $0.043 \mathrm{~g}$ (0.226 mmol) of $\mathrm{EDC} \cdot \mathrm{HCl}, 0.014 \mathrm{~g}(0.113 \mathrm{mmol})$ of DMAP and $30 \mathrm{ml}$ of distilled $\mathrm{CH}_{2} \mathrm{Cl}_{2}$ to afford $0.090 \mathrm{~g}$ of $\mathbf{L}^{8}$ as a white powder (73\%). ${ }^{1} \mathrm{H}$ NMR $\left(\mathrm{CDCl}_{3}\right) \delta 0.87\left(\mathrm{~m}, 12 \mathrm{H}, \mathrm{CH}_{3}\right), 1.25\left(\mathrm{br}, 40 \mathrm{H},\left(\mathrm{CH}_{2}\right)_{5}\right)$, $1.70\left(\mathrm{t},{ }^{3} J=6.7 \mathrm{~Hz}, 8 \mathrm{H}, \mathrm{CH}_{2}\right), 2.14$ (s, 3H, $\left.\mathrm{CH}_{3}\right), 2.86$ (s, 3H, $\mathrm{CH}_{3}$ phen.), 3.89 (t, ${ }^{3} J=6.7 \mathrm{~Hz}$, $\left.8 \mathrm{H}, \mathrm{OCH}_{2}\right), 5.68$ (s, 2H, $\mathrm{OCH}_{2}$ phen.), $6.56\left(\mathrm{t},{ }^{4} J=2.1 \mathrm{~Hz}, 2 \mathrm{H}, \mathrm{H}\right.$ arom. $), 7.06\left(\mathrm{~d},{ }^{4} J=2.1 \mathrm{~Hz}\right.$, 4H, H arom.), 7.48 (d, ${ }^{3} \mathrm{~J}=7.3 \mathrm{~Hz}, 1 \mathrm{H}, \mathrm{H}$ phen.), 7.68 (m, 3H, H phen.), 8.11 (m, 3H, H phen. and arom.), 8.44 (br, 2H, NH). ${ }^{13} \mathrm{C}$ NMR $\left(\mathrm{CDCl}_{3}\right) \delta 13.9,14.1,22.6,25.6,26.0,29.2,29.4$, 
29.7, 31.8 (alkyl chains and $\left.2 \mathrm{CH}_{3}\right), 68.1,68.3\left(\mathrm{OCH}_{2}\right.$ alkyl chains and $\mathrm{OCH}_{2}$ phen.), 105.2, $105.7,121.3,123.8,125.4,126.5,127.0,128.1,133.0,136.1,136.4,136.7,137.2,145.2$, 156.4, 159.5, 160.5 (phenyl and phen.), 165.3, 166.1 (amide and ester). $\mathrm{FAB}^{+} \mathrm{m} / \mathrm{z}$ (nature of the peak, relative intensity) $1093.2\left([\mathrm{M}+\mathrm{H}]^{+}, 100\right), 716.2\left(\left[\mathrm{M}-\mathrm{C}_{23} \mathrm{H}_{38} \mathrm{NO}_{3}+\mathrm{H}\right]^{+}, 30\right)$. FT-IR (KBr cell, cm $\left.{ }^{-1}\right) 3400$ (sh), 3247 (s), 2926 (s), 2855 (s), 1734 (m), 1650 (sh), 1644 (s), 1594 (vs), 1525 (sh), 1509 (s), 1444 (s), 1351 (m), 1320 (m), 1166 (s), 1056 (m). Anal. Calc. for $\mathrm{C}_{68} \mathrm{H}_{92} \mathrm{~N}_{4} \mathrm{O}_{8} \cdot \mathrm{H}_{2} \mathrm{O}: \mathrm{C}, 73.48 ; \mathrm{H}, 8.52 ; \mathrm{N}, 5.04$. Found: C, 73.15; H, 8.27; N, 4.75.

Ligand $\mathbf{L}^{\mathbf{1 2}}$. The compound was prepared from $0.100 \mathrm{~g}(0.09 \mathrm{mmol})$ of $\mathbf{1}^{\mathbf{1 2}}, 0.020 \mathrm{~g}(0.09$ mmol) of 2-(hydroxymethyl)-9-methyl-1,10-phenanthroline, $0.034 \mathrm{~g} \quad(0.18 \mathrm{mmol})$ of $\mathrm{EDC} \cdot \mathrm{HCl}, 0.011 \mathrm{~g}(0.09 \mathrm{mmol})$ of DMAP and $30 \mathrm{~mL}$ of distilled $\mathrm{CH}_{2} \mathrm{Cl}_{2}$ to afford $0.090 \mathrm{~g}$ of $\mathbf{L}^{\mathbf{1 2}}$ as a white powder (76\%). ${ }^{1} \mathrm{H}$ NMR $\left(\mathrm{CDCl}_{3}\right) \delta 0.87\left(\mathrm{~m}, 12 \mathrm{H}, \mathrm{CH}_{3}\right), 1.25$ (br, $72 \mathrm{H}$, $\left.\left(\mathrm{CH}_{2}\right)_{9}\right), 1.73$ (t, $\left.{ }^{3} J=7.2 \mathrm{~Hz}, 8 \mathrm{H}, \mathrm{CH}_{2}\right), 2.18$ (s, 3H, $\left.\mathrm{CH}_{3}\right), 2.89$ (s, 3H, $\mathrm{CH}_{3}$ phen.), 3.92 (t, ${ }^{3} J$ $\left.=6.4 \mathrm{~Hz}, 8 \mathrm{H}, \mathrm{OCH}_{2}\right), 5.75\left(\mathrm{~s}, 2 \mathrm{H}, \mathrm{OCH}_{2}\right.$ phen. $), 6.58\left(\mathrm{t},{ }^{4} J=2.1 \mathrm{~Hz}, 2 \mathrm{H}, \mathrm{H}\right.$ arom.) $7.04\left(\mathrm{~d},{ }^{4} J\right.$ $=2.1 \mathrm{~Hz}, 4 \mathrm{H}, \mathrm{H}$ arom.), 7.49 (d, ${ }^{3} J=8.0 \mathrm{~Hz}, 1 \mathrm{H}, \mathrm{H}$ phen.), 7.69 (m, 3H, H phen.), 8.14 (m, $3 \mathrm{H}, \mathrm{H}$ phen. and arom.), 8.23 (br, $2 \mathrm{H}, \mathrm{NH}) .{ }^{13} \mathrm{C} \mathrm{NMR}\left(\mathrm{CDCl}_{3}\right) \delta 14.12,14.39,22.97,25.91$, 26.27, 29.47, 29.62, 29.67, 29.91, 29.96, 32.20, 68.43, $68.67\left(\mathrm{OC}_{12}+2 \mathrm{CH}_{3}\right), 105.50,105.98$, $121.56,124.10,125.74,126.76,127.26,128.44,133.13,136.45,136.64,136.96,137.46$, $145.45,145.60,156.73,159.83,160.80$ (phenyl and phen), 165.57, 166.35 (amide and ester). $\mathrm{FAB}^{+} \mathrm{m} / \mathrm{z}$ (nature of the peak, relative intensity) 1317.2([M+H] $\left.]^{+}, 100\right), 828.1$ ([M$\left.\left.\mathrm{C}_{31} \mathrm{H}_{54} \mathrm{NO}_{3}+\mathrm{H}\right]^{+}, 10\right)$. FT-IR (KBr cell, $\mathrm{cm}^{-1}$ ) 3400 (sh), 3234 (m), 2923 (vs), 2853 (s), 1721 (m), 1655 (shoulder), $1645(\mathrm{~s}), 1594$ (vs), 1525 (sh), 1505 (m), 1446 (s), 1385 (w), 1350 (w), 1324 (m), 1259 (w), 1217 (w), 1166 (s), 1108 (w), 1057 (m). Anal. Calc. for $\mathrm{C}_{84} \mathrm{H}_{124} \mathrm{~N}_{4} \mathrm{O}_{8} \cdot \mathrm{H}_{2} \mathrm{O}: \mathrm{C}, 75.52 ; \mathrm{H}, 9.51 ; \mathrm{N}, 4.19$. Found: C, 75.36; H, 9.15; N, 4.09.

Ligand $\mathbf{L}^{\mathbf{1 6}}$. The compound was prepared from $0.150 \mathrm{~g}(0.113 \mathrm{mmol})$ of $\mathbf{1}^{\mathbf{1 6}}, 0.025 \mathrm{~g}(0.113$ mmol) of 2-(hydroxymethyl)-9-methyl-1,10-phenanthroline, $0.043 \mathrm{~g}(0.226 \mathrm{mmol})$ of $\mathrm{EDC} \cdot \mathrm{HCl}, 0.014 \mathrm{~g}(0.113 \mathrm{mmol})$ of DMAP and $30 \mathrm{~mL}$ of distilled $\mathrm{CH}_{2} \mathrm{Cl}_{2}$ to afford $0.13 \mathrm{~g}$ of $\mathbf{L}^{\mathbf{1 6}}$ as a white powder $(75 \%) .{ }^{1} \mathrm{H}$ NMR $\left(\mathrm{CDCl}_{3}\right) \delta 0.87\left(\mathrm{~m}, 12 \mathrm{H}, \mathrm{CH}_{3}\right), 1.25(\mathrm{br}, 104 \mathrm{H}$, 
$\left.\left(\mathrm{CH}_{2}\right)_{13}\right), 1.71\left(\mathrm{~m}, 8 \mathrm{H}, \mathrm{CH}_{2}\right), 2.14$ (s, 3H, $\left.\mathrm{CH}_{3}\right), 2.86$ (s, 3H, $\mathrm{CH}_{3}$ phen.), 3.90 (t, ${ }^{3} \mathrm{~J}=6.4 \mathrm{~Hz}$, $8 \mathrm{H}, \mathrm{OCH}_{2}$ ), 5.69 (s, 2H, $\mathrm{OCH}_{2}$ phen.), 6.56 (s, 2H, H arom.), $7.06\left(\mathrm{~d},{ }^{4} \mathrm{~J}=2.1 \mathrm{~Hz}, 4 \mathrm{H}, \mathrm{H}\right.$ arom.), 7.48 (d, ${ }^{3} J=8.0 \mathrm{~Hz}, 1 \mathrm{H}, \mathrm{H}$ phen.), 7.60 (m, 3H, H phen.), 8.10 (m, 3H, H phen. and arom.), 8.43 (br, 2H, NH). ${ }^{13} \mathrm{C} \mathrm{NMR}\left(\mathrm{CDCl}_{3}\right) \delta 13.9,14.1,22.7,25.6,26.0,29.2,29.4,29.7$, 31.9 (alkyl chains and $\left.2 \mathrm{CH}_{3}\right), 68.1,68.3\left(\mathrm{OCH}_{2}\right.$ alkyl chains and $\mathrm{OCH}_{2}$ phen.), 105.2, 105.7, $121.3,123.8,125.5,126.5,126.9,128.1,132.9,136.1,136.4,136.7,137.2,145.2,156.4$, $159.5,160.5$ (phenyl and phen.), 165.3, 166.1 (amide and ester). $\mathrm{FAB}^{+} \mathrm{m} / \mathrm{z}$ (nature of the peak, relative intensity) $1542.2\left([\mathrm{M}+\mathrm{H}]^{+}, 100\right), 940.4\left(\left[\mathrm{M}-\mathrm{C}_{39} \mathrm{H}_{70} \mathrm{NO}_{3}+\mathrm{H}\right]^{+}, 25\right)$. FT- IR $(\mathrm{KBr}$ cell, $\mathrm{cm}^{-1}$ ) 3400 (sh), 3224 (m), 2919 (vs), 2850 (s), 1720 (m), 1655 (sh), 1645 (s), 1593 (s), 1520 (sh), 1508 (m), 1465 (m), 1442 (m), 1321 (m), 1256 (w), 1190 (vw), 1168 (s). Anal. Calc. for $\mathrm{C}_{100} \mathrm{H}_{156} \mathrm{~N}_{4} \mathrm{O}_{8} \cdot \mathrm{H}_{2} \mathrm{O}: \mathrm{C}, 76.98 ; \mathrm{H}, 10.21 ; \mathrm{N}, 3.59$. Found: C, 76.69; H, 10.05; N, 3.38.

General procedure for the preparation of the complex $\left[\mathrm{Cu}\left(\mathbf{L}^{\mathbf{n}}\right)_{2}\right] \mathrm{BF} \mathrm{F}_{4}$ : A Schlenk flask equipped with a septum and an argon inlet was charged with the ligand $\mathbf{L}^{\mathbf{n}}$ (1 equiv.), distilled $\mathrm{CH}_{2} \mathrm{Cl}_{2}$ and $\left[\mathrm{Cu}\left(\mathrm{CH}_{3} \mathrm{CN}\right)_{4}\right] \mathrm{BF}_{4}$ (0.5 equiv.). The solution was stirred during $1 \mathrm{~h}$. After evaporation to dryness $\mathrm{CH}_{2} \mathrm{Cl}_{2}$ was added, and the insoluble material filtered out. Purification was performed by slow evaporation of $\mathrm{CH}_{2} \mathrm{Cl}_{2}$ from $\mathrm{CH}_{2} \mathrm{Cl}_{2} / \mathrm{CH}_{3} \mathrm{CN}$ mixture, followed by a subsequent precipitation in a $\mathrm{CH}_{2} \mathrm{Cl}_{2} / \mathrm{CH}_{3} \mathrm{OH}$ mixture.

Complex $\left[\mathrm{Cu}\left(\mathbf{L}^{\mathbf{1}}\right)_{2}\right] \mathrm{BF}_{4}$ : The compound was prepared from $0.074 \mathrm{~g}(0.105 \mathrm{mmol})$ of $\mathbf{L}^{1}, 0.020 \mathrm{~g}(0.063 \mathrm{mmol})$ of $\left[\mathrm{Cu}\left(\mathrm{CH}_{3} \mathrm{CN}\right)_{4}\right] \mathrm{BF}_{4}$ and distilled $\mathrm{CH}_{2} \mathrm{Cl}_{2}(10 \mathrm{~mL})$ to afford 0.080 g of a deep-red complex (81\%). ${ }^{1} \mathrm{H}$ NMR $\left(200 \mathrm{MHz}, \mathrm{CDCl}_{3}\right) \delta 2.27\left(\mathrm{~s}, 6 \mathrm{H}, \mathrm{CH}_{3}\right), 2.63$ (s, $6 \mathrm{H}, \mathrm{CH}_{3}$ phen), $3.76\left(\mathrm{~s}, 24 \mathrm{H}, 4 \mathrm{OCH}_{3}\right), 4.75\left(\mathrm{AB}\right.$ quartet, $J_{A B}=11.7 \mathrm{~Hz}, \Delta \mathrm{v}=24.0 \mathrm{~Hz}, 4 \mathrm{H}$, $\mathrm{OCH}_{2}$ phen), 6.57 (t, ${ }^{3} \mathrm{~J}=2.2 \mathrm{~Hz}, 4 \mathrm{H}, \mathrm{H}$ arom.), 7.03 (d, ${ }^{3} \mathrm{~J}=2.2 \mathrm{~Hz}, 8 \mathrm{H}, \mathrm{H}$ arom.), 7.36 (s, 4H, H arom.), 7.77 (d, ${ }^{3} J=8.4 \mathrm{~Hz}, 4 \mathrm{H}, \mathrm{H}$, phen), 7.93 (m, 4H, H phen), 8.39 (d, ${ }^{3} J=7.6 \mathrm{~Hz}$, $2 \mathrm{H}, \mathrm{H}$ phen), $8.46\left(\mathrm{~d},{ }^{3} \mathrm{~J}=8.4 \mathrm{~Hz}, 2 \mathrm{H}, \mathrm{H}\right.$ phen), 8.64 (br, $4 \mathrm{H}, \mathrm{NH}$ ). $\mathrm{FAB}^{+} m / z$ (nature of the peak, relative intensity) $1463.2\left(\left[\mathrm{M}-B F_{4}\right]^{+}, 100\right)$. FT-IR $\left(\mathrm{KBr}\right.$ cell, $\left.\mathrm{cm}^{-1}\right) 3230(\mathrm{~m}), 2952(\mathrm{w})$, 2836 (w), 1720 (m), 1658 (m), 1595 (vs), 1513 (s), 1456 (s), 1323 (s), 1207 (s), 1155 (s), 
1080 (s). Anal. Calc. for $\mathrm{C}_{80} \mathrm{H}_{72} \mathrm{~N}_{8} \mathrm{O}_{16} \mathrm{CuBF}_{4}: \mathrm{C}, 61.92 ; \mathrm{H}, 4.68 ; \mathrm{N}, 7.22$. Found: $\mathrm{C}, 61.70 ; \mathrm{H}$, $4.35 ; \mathrm{N}, 6.99$.

Complex $\left[\mathrm{Cu}\left(\mathbf{L}^{\mathbf{1}}\right)_{2}\right] \mathrm{ClO}_{4}$ : The compound was prepared from $0.100 \mathrm{~g}(0.142 \mathrm{mmol})$ of $\mathbf{L}^{\mathbf{1}}$, $0.025 \mathrm{~g}(0.072 \mathrm{mmol})$ of $\left[\mathrm{Cu}\left(\mathrm{CH}_{3} \mathrm{CN}\right)_{4}\right] \mathrm{ClO}_{4}$ and distilled $\mathrm{CH}_{2} \mathrm{Cl}_{2}(15 \mathrm{~mL})$ to afford $0.205 \mathrm{~g}$ of a deep-red complex (92\%). ${ }^{1} \mathrm{H}$ NMR similar to the $\left[\mathrm{Cu}\left(\mathbf{L}^{\mathbf{1}}\right)_{2}\right] \mathrm{BF}_{4}$ complex. $\mathrm{FAB}^{+} \mathrm{m} / \mathrm{z}$ (nature of the peak, relative intensity) 1463.1([M-ClO 4$\left.]^{+}, 100\right)$. FT-IR $\left(\mathrm{KBr}\right.$ cell, $\left.\mathrm{cm}^{-1}\right) 3229$ (m), 1723 (m), 1660 (m), 1598 (s), 1515 (s), 1454 (s), 1323 (s), 1210 (s), 1154 (s), 1100 (s), 1080 (s), 852 (m). Anal. Calc. for $\mathrm{C}_{80} \mathrm{H}_{72} \mathrm{~N}_{8} \mathrm{O}_{16} \mathrm{CuClO}_{4}$ : C, 61.42; H, 4.64; N, 7.16. Found: C, 61.28; H, 4.46; N, 7.07.

Complex $\left[\mathrm{Cu}\left(\mathbf{L}^{\mathbf{8}}\right)_{2}\right] \mathrm{BF}_{4}$ : The compound was prepared from $0.055 \mathrm{~g}(0.050 \mathrm{mmol})$ of $\mathbf{L}^{\mathbf{8}}$, $0.008 \mathrm{~g}(0.025 \mathrm{mmol})$ of $\left[\mathrm{Cu}\left(\mathrm{CH}_{3} \mathrm{CN}\right)_{4}\right] \mathrm{BF}_{4}$ and distilled $\mathrm{CH}_{2} \mathrm{Cl}_{2}(7 \mathrm{~mL})$ to afford $0.058 \mathrm{~g}$ of a deep-red complex $(99 \%) .{ }^{1} \mathrm{H}$ NMR $\left(200 \mathrm{MHz}, \mathrm{CDCl}_{3}\right) \delta 0.87\left(\mathrm{~m}, 24 \mathrm{H}, 6 \mathrm{CH}_{3}\right), 1.26$ (br, 80H, $\left.\left(\mathrm{CH}_{2}\right)_{5}\right), 1.73$ (m, 16H, $\left.\mathrm{CH}_{2}\right), 2.29$ (s, 6H, $\left.\mathrm{CH}_{3}\right), 2.66$ (s, 6H, $\mathrm{CH}_{3}$ phen), 3.90 (m, 16H, $\mathrm{OCH}_{2}$ alkyl chains), 4.75 (AB quartet, $J_{A B}=10.9 \mathrm{~Hz}, \Delta \mathrm{v}=24.4 \mathrm{~Hz}, 4 \mathrm{H}, \mathrm{OCH}_{2}$ phen), 6.57 (s, 4H, H arom.), 7.00 (s, 8H, H arom.), 7.39 (s, 4H, H arom.), 7.78 (m, 4H, H, phen), 7.93 (m, 4H, H phen), 8.37 (d, ${ }^{3} \mathrm{~J}=8.0 \mathrm{~Hz}, 2 \mathrm{H}, \mathrm{H}$ phen), 8.48 (d, ${ }^{3} \mathrm{~J}=8.0 \mathrm{~Hz}, 2 \mathrm{H}, \mathrm{H}$ phen), 8.55 (br, $4 \mathrm{H}, \mathrm{NH}$ ). $\mathrm{FAB}^{+} \mathrm{m} / \mathrm{z}$ (nature of the peak, relative intensity) $2249.2\left(\left[\mathrm{M}-\mathrm{BF}_{4}\right]^{+}, 30\right), 1155.2$ $\left(\left[\mathrm{M}-\mathrm{L}^{8}-\mathrm{BF}_{4}\right]^{+}, 100\right)$. FT-IR (KBr cell, cm $\left.{ }^{-1}\right) 3280$ (m), 2926 (vs), 2856 (s), 1723 (m), 1651 (m), 1593 (vs), 1512 (m), 1445 (m), 1324 (m), 1260 (w), 1163 (vs), 1055 (vs). Anal. Calc. for $\mathrm{C}_{136} \mathrm{H}_{184} \mathrm{~N}_{8} \mathrm{O}_{16} \mathrm{CuBF}_{4}: \mathrm{C}, 69.89 ; \mathrm{H}, 7.93 ; \mathrm{N}, 4.79$. Found: C, 69.51; H, 7.60; N, 4.40.

Complex $\left[\mathrm{Cu}\left(\mathbf{L}^{\mathbf{1 2}}\right)_{2}\right] \mathrm{BF}_{4}$ : The compound was prepared from $0.15 \mathrm{~g}(0.114 \mathrm{mmol})$ of $\mathbf{L}^{\mathbf{1 2}}$, $0.018 \mathrm{~g}(0.057 \mathrm{mmol})$ of $\left[\mathrm{Cu}\left(\mathrm{CH}_{3} \mathrm{CN}\right)_{4}\right] \mathrm{BF}_{4}$ and distilled $\mathrm{CH}_{2} \mathrm{Cl}_{2}(30 \mathrm{~mL})$ to afford $0.109 \mathrm{~g}$ of a deep-red complex $(68 \%)$. ${ }^{1} \mathrm{H}$ NMR $\left(200 \mathrm{MHz}, \mathrm{CDCl}_{3}\right) \delta 0.87\left(\mathrm{~m}, 24 \mathrm{H}, 6 \mathrm{CH}_{3}\right), 1.25(\mathrm{br}$, 144H, $\left.\left(\mathrm{CH}_{2}\right)_{9}\right), 1.72\left(\mathrm{~m}, 16 \mathrm{H}, \mathrm{CH}_{2}\right), 2.30$ (s, 6H, CH 3 ), 2.66 (s, 6H, $\mathrm{CH}_{3}$ phen), 3.90 (m, 16H, $\mathrm{OCH}_{2}$ alkyl chains), 4.74 (AB quartet, $J_{A B}=10.8 \mathrm{~Hz}, \Delta v=24.4 \mathrm{~Hz}, 4 \mathrm{H}, \mathrm{OCH}_{2}$ phen), $6.56(\mathrm{~s}$, 4H, H arom.), 7.00 (d, ${ }^{3} \mathrm{~J}=2.2 \mathrm{~Hz}, 8 \mathrm{H}, \mathrm{H}$ arom.), 7.38 (s, 4H, H arom.), 7.77 (d, ${ }^{3} J=8.4 \mathrm{~Hz}$, 2H, H, phen), 7.79 (d, ${ }^{3} J=8.4 \mathrm{~Hz}, 2 \mathrm{H}, \mathrm{H}$ phen), 7.92 (m, 4H, H phen), 8.37 (d, ${ }^{3} J=7.7 \mathrm{~Hz}$, 
2H, H phen), 8.48 (d, ${ }^{3} J=8.4 \mathrm{~Hz}, 2 \mathrm{H}, \mathrm{H}$ phen), 8.54 (br, $\left.4 \mathrm{H}, \mathrm{NH}\right) .{ }^{13} \mathrm{C} \mathrm{NMR}\left(\mathrm{CDCl}_{3}\right) \delta 13.6$, 14.1, 22.7, 26.1, 26.6, 29.3, 29.4, 29.5, 29.7, 29.7, 29.7, 32.0, 68.4 (Aakyl chains, 2 $\mathrm{CH}_{3}$, $\mathrm{OCH}_{2}$ phen) 105.6, 123.2, 125.6, 125.7, 126.8, 126.9, 127.8, 129.3, 136.1, 136.5, 137.5, 138.7, 138.8, 143.3, 153.1, 153.1, 157.3 (H arom., H phen.) 160.5, 166.0 (amide, ester). FAB ${ }^{+}$ $m / z$ (nature of the peak, relative intensity) $2697.2\left(\left[\mathrm{M}-\mathrm{BF}_{4}\right]^{+}, 100\right), 1379.2\left(\left[\mathrm{M}-\mathrm{L}^{12}-\mathrm{BF}_{4}\right]^{+}\right.$, 30). FT-IR (KBr cell, cm ${ }^{-1}$ ) 3288 (m), 2924 (vs), 2854 (s), 1723 (m), 1652 (m), 1594 (s), 1513 (m), 1445 (m), 1324 (m), 1162 (s), 1056 (s). Anal. Calc. for $\mathrm{C}_{168} \mathrm{H}_{248} \mathrm{~N}_{8} \mathrm{O}_{16} \mathrm{CuBF}_{4}$ : C, 72.42; H, 8.97; N, 4.02. Found: C, 72.19; H, 8.68; N, 3.83.

Complex $\left[\mathrm{Cu}\left(\mathbf{L}^{\mathbf{1 6}}\right)_{2}\right] \mathrm{BF}_{4}$ : The compound was prepared from $0.200 \mathrm{~g}(0.129 \mathrm{mmol})$ of $\mathbf{L}^{\mathbf{1 6}}$, $0.021 \mathrm{~g}(0.065 \mathrm{mmol})$ of $\left[\mathrm{Cu}\left(\mathrm{CH}_{3} \mathrm{CN}\right)_{4}\right] \mathrm{BF}_{4}$ and distilled $\mathrm{CH}_{2} \mathrm{Cl}_{2}(40 \mathrm{~mL})$ to afford $0.200 \mathrm{~g}$ of deep-red complex (95\%). ${ }^{1} \mathrm{H}$ NMR $\left(200 \mathrm{MHz}, \mathrm{CDCl}_{3}\right) \delta 0.87\left(\mathrm{~m}, 24 \mathrm{H}, 6 \mathrm{CH}_{3}\right), 1.25(\mathrm{br}$, 208H, $\left.\left(\mathrm{CH}_{2}\right)_{13}\right), 1.72\left(\mathrm{~m}, 16 \mathrm{H}, \mathrm{CH}_{2}\right), 2.29$ (s, 6H, $\left.\mathrm{CH}_{3}\right), 2.66$ (s, 6H, $\mathrm{CH}_{3}$ phen), 3.89 (m, 16H, $\mathrm{OCH}_{2}$ alkyl chains), $4.74\left(\mathrm{AB}\right.$ quartet, $J_{A B}=10.9 \mathrm{~Hz}, \Delta \mathrm{v}=24.4 \mathrm{~Hz}, 4 \mathrm{H}, \mathrm{OCH}_{2}$ phen), 6.56 (s, 4H, H arom.), 6.99 (s, 8H, H arom.), 7.38 (s, 4H, H arom.), 7.78 (m, 4H, H, phen), 7.94 (m, 4H, H phen), 8.37 (d, ${ }^{3} \mathrm{~J}=8.0 \mathrm{~Hz}, 2 \mathrm{H}, \mathrm{H}$ phen), 8.48 (d, ${ }^{3} \mathrm{~J}=8.4 \mathrm{~Hz}, 2 \mathrm{H}, \mathrm{H}$ phen), 8.54 (br, $4 \mathrm{H}, \mathrm{NH}$ ). $\mathrm{FAB}^{+} \mathrm{m} / \mathrm{z}$ (nature of the peak, relative intensity) $3146.3\left(\left[\mathrm{M}-\mathrm{BF}_{4}\right]^{+}, 40\right), 1604.2$ $\left(\left[\mathrm{M}-\mathrm{L}^{16}-\mathrm{BF}_{4}\right]^{+}, 100\right)$. FT-IR (KBr cell, $\left.\mathrm{cm}^{-1}\right), 3285$ (m), 2924 (vs), 2854 (s), 1723 (m), 1652 (m), 1594 (s), 1510 (m), 1455 (m), 1445 (m), 1324 (m), 1163 (s), 1054 (s). Anal. Calc. for $\mathrm{C}_{200} \mathrm{H}_{312} \mathrm{~N}_{8} \mathrm{O}_{16} \mathrm{CuBF}_{4}: \mathrm{C}, 74.25 ; \mathrm{H}, 9.72 ; \mathrm{N}, 3.46$. Found: C, 73.89; H, 9.35; N, 3.00.

\section{X-ray structure determinations.}

Crystal data for compound $\mathrm{L}^{1}: \mathrm{C}_{40} \mathrm{H}_{36} \mathrm{~N}_{4} \mathrm{O}_{8}, \mathrm{M}=700.76 \mathrm{~g}$, triclinic, space group P-1, $\mathrm{a}=$ 8.974(2), $b=12.086(2), c=17.068$ ( 3) $\AA, \alpha=68.87(5), \beta=80.04(5), \gamma=79.18(5))^{\circ}, V=$ 1684.8(6) $\AA^{3}, \mathrm{Z}=2, \mathrm{Dc}=1.381 \mathrm{gcm}^{-3}, \lambda=0.966 \AA, \mathrm{T}=100(2)^{\circ} \mathrm{K}, \mu_{0.966}=0.097 \mathrm{~mm}^{-1}, \mathrm{~F}(000)$ $=736$. Crystals of ligand $\mathrm{L}^{1}$ were of too small dimensions to give sufficient diffraction intensities on CCD or standard diffractometers. Microcrystals (100x50x25 microns) were transferred in a drop of paraffin, oil was isolated with a $0.2 \mathrm{~mm}$ cryoloop and fitted on the MAR Research Image Plate MAR345 at the W32 beam-line station of the LURE DCI synchrotron (Orsay, France). ${ }^{1}$ The temperature was set to $100 \mathrm{~K}$ using an Oxford Cryocooling 
set up. Data recording consisted of 79 frames (exposure time $=10 \mathrm{mn}$ for 3 degree rotation each) at the nominal resolution of $0.97 \AA$. Indexing and processing of the frames were done with the MOSFLM program, ${ }^{2}$ associated with the CCP4 suite of programs ${ }^{3}$ to derive the structure factors. A total of 12525 reflections were processed and reduced to 3506 independent reflections $\left(\mathrm{R}_{\text {equiv }}=5.2 \%\right)$. The structure was solved by direct methods [SHELXS86] ${ }^{4}$ and refined anisotropically on $\mathrm{F}^{2}$ for all reflections by least-squares methods using [SHELXL-93] ${ }^{5}$. All hydrogen atoms were located on difference- Fourier syntheses. They were included in the refinement at their ideal positions $\left(\mathrm{C}-\mathrm{H}_{\mathrm{ar}}=0.93 \AA, \mathrm{C}-\mathrm{H}_{\mathrm{Me}}=0.96 \AA\right)$ and assigned an isotropic thermal parameter of 1.2 that of the bonded atoms, whereas the hydrogen atoms of the amine groups, located from the $\Delta \rho$ were fixed during the subsequent calculations. The final conventional $R$ factor was 0.055 for 3504 data and 475 parameters, $\left.\mathrm{wR}\left(F^{2}\right)=0.146, \mathrm{w}=1 /\left[\sigma^{2}\left(F o^{2}\right)+(0.0879) P\right)^{2}+2.05\right]$, where $\mathrm{P}=\left(F o^{2}+2 F c^{2}\right) / 3$. The largest difference peak and hole are respectively 0.51 and $-0.22 \mathrm{e} / \AA^{3}$. 
Crystal data of $\left[\mathrm{Cu}\left(\mathrm{L}^{\mathbf{1}}\right)_{2}\right] \mathrm{ClO}_{4}:\left[\mathrm{Cu}(\mathrm{I}) \mathrm{C}_{80} \mathrm{~N}_{8} \mathrm{O}_{16} \mathrm{H}_{72}\right]\left(\mathrm{ClO}_{4}\right), 4 \mathrm{CH}_{3} \mathrm{CN}, \mathrm{M}=1728.66$, triclinic, space group P-1. Cell parameters are: $a=15.890(3) \AA ; b=16.327(3) \AA ; c=17.777(3) \AA, \alpha=$ $79.50(5)^{\circ} ; \beta=72.02(5)^{\circ}, \gamma=73.90(5)^{\circ} ; \mathrm{V}=4191 \AA^{3}, Z=2, \lambda=0.982 \AA, d_{c}=1.370 \mathrm{gcm}^{-3}$, $\mathrm{F}(000)=1804, \mu=0.373 \mathrm{~mm}^{-1}$. Crystallization of the phenanthroline $/ \mathrm{Cu}(\mathrm{I})$ compound gave micro prismatic shaped rods, about 50 micron thick and 150 microns long. A single micro crystal randomly oriented was used for the complete X-ray diffraction data recording at the DW-32 station on the wiggler beamline at the synchrotron DCI (Orsay) using a MAR345 image plate system at $\lambda=0.982 \AA .{ }^{1}$ As the crystals were unstable once removed from their mother liquor, the cryo-cooling technique was employed using a nylon loop to rapidly anneal a crystal at $177 \mathrm{~K}\left(-94^{\circ} \mathrm{C}\right)$. More than a full $360^{\circ}$ rotation range was recorded (100 frames 4degree rotation each). Due to geometric restrictions, the resolution at the edge of the detector was set as $1.01 \AA$. Data reductions were done using the DENZO program from the HKL suite. ${ }^{6}$ Crystals of phenanthroline/Cu(I) are of bad quality and highly mosaic. They also usually carry satellites that give additional reflections out of the main lattice. This allowed to integrate 30285 reflections which were subsequently reduced to 7059 independent reflections $\left(\mathrm{R}_{\text {merge }}=0.045\right)$ and formatted in a suitable form for the SHELXL suite of programs. ${ }^{5}$ The final data set consists of 7059 reflections of which 5965 are considered as observed (with $F_{o} \geq$ $\left.2 \sigma \mathrm{F}_{\mathrm{o}}\right)$.

The structure was solved by direct methods (SHELXS-86) ${ }^{4}$ and was refined on $\mathrm{F}^{2}$ for all reflections by least-squares methods using SHELXL-93. ${ }^{5}$ Only the copper, chlorine, oxygen and nitrogen atoms were refined anisotropically as to limit the number of parameters versus the number of data. Hydrogen atoms were modeled at their theoretical positions $\left(\mathrm{C}-\mathrm{H}_{\mathrm{ar}}=\right.$ $0.93 \AA, \mathrm{C}-\mathrm{H}_{\mathrm{Me}}=0.96 \AA$ ) using an isotropic thermal factor equal to 1.2 times that of the bonded atom and introduced in the refinement cycles. The final conventional $\mathrm{R}$ is 0.1011 for $5965 \mathrm{Fo}>4 \sigma(\mathrm{Fo})$ and 675 parameters, and 0.1138 for all data, $\mathrm{wR}\left(\mathrm{F}^{2}\right)=0.26$ for all, $\mathrm{w}=1 /$ $\left[\sigma^{2}(\mathrm{Fo})^{2}+(0.1615 \mathrm{P})^{2}+24.20 \mathrm{P}\right]$ where $\mathrm{P}=\left(\mathrm{Fo}^{2}+2 \mathrm{Fc}^{2}\right) / 3$. The largest difference peak and hole are 0.79 and $-0.65 \mathrm{e}^{-3}$.

Gelation test: A weighted amount of the compound was mixed with a determined volume of the solvent in a septum-capped test tube. The mixture was heated until the solid was completely dissolved. The resulting clear solution was cooled down in air to $25^{\circ} \mathrm{C}$, than left 
overnight to complete the gelation. The gelation kinetics depends on the concentration from minute to hours. The aspect of the mixture was then examined by turning the test tube upsidedown.

POM, DSC and XRD measurements of the mesomorphic materials. The optical textures of the mesophases were studied with a Leitz polarizing microscope equipped with a Mettler FP80 hot-stage and an FP80 central processor. The transition temperatures and enthalpies were measured by differential scanning calorimetry with a Perkin-Elmer DSC-7 instrument operated at a scanning rate of $10{ }^{\circ} \mathrm{C} \min ^{-1}$ on heating. The apparatus was calibrated with indium $\left(156.6{ }^{\circ} \mathrm{C} ; 28.4 \mathrm{Jg}^{-1}\right)$ and gallium $\left(29.8^{\circ} \mathrm{C}\right)$ as the standards. The TGA measurements were carried out on a SDTQ 600 apparatus at scanning rate of $10{ }^{\circ} \mathrm{C} \mathrm{min}^{-1}$. The XRD patterns were obtained with two different experimental set-ups. In all cases, a linear monochromatic $\mathrm{Cu}-\mathrm{K} \alpha_{1}$ beam $(\lambda=1.5405 \AA)$ was obtained using a sealed-tube generator (900 W) equipped with a bent quartz monochromator. In the first set, the transmission Guinier geometry was used, whereas a Debye-Scherrer-like geometry was used in the second experimetal set-up. In all cases, the crude powder was filled in Lindemann capillaries of $1 \mathrm{~mm}$ diameter. An initial set of diffraction patterns was recorded on an image plate; periodicities up to $80 \AA$ can be measured, and the sample temperature controlled to within $\pm 0.3{ }^{\circ} \mathrm{C}$. The second set of diffraction patterns was recorded with a curved Inel CPS 120 counter gas-filled detector linked to a data acquisition computer; periodicites up to $60 \AA$ can be measured, and the sample temperature controlled to within $\pm 0.05{ }^{\circ} \mathrm{C}$. In each case, exposure times were varied from 1 to $24 \mathrm{~h}$.

\section{References}

(1) R. Fourme, P. Dhez, J.-P. Benoit, R. Khan, J.-M. Dubuisson, P. Besson, and J. Frouin, Rev. Sci. Instrum., 1992, 63, 982.

(2) A. Leslie, MOSFLM User Guide, version 5.20, MRC Laboratory of Molecular Bioiogy, Cambridge, United Kingdom, 1994. 
(3) CCP4 - Collaborative Computational Project, Number 4. The CCP4 suite: Programs for protein crystallography. Acta Crystallogr. 1994, D50, 760.

(4) G. M. Sheldrick, Acta Crystallogr., Sec A. 1990, 46, 467.

(5) G. M. Sheldrick, SHELX-93, Program for the Refinement of Crystal Structures, University of Gottingen, Germany, 1993.

(6) Otwinowski, Z. and Minor, W. "Processing of X-ray data". Methods in Enzymol. 1997, 276, 307 . 